\title{
Antimicrobial use, drug-resistant infections and COVID-19
}

Timothy M. Rawson, Damien Ming (1), Raheelah Ahmad, Luke S. P. Moore (i) and Alison H. Holmes ${ }^{\bowtie}$

Coronavirus disease 2019 may have a complex long-term impact on antimicrobial resistance (AMR). Coordinated strategies at the individual, health-care and policy levels are urgently required to inform necessary actions to reduce the potential longer-term impact on AMR and on access to effective antimicrobials.

Coronavirus disease 2019 (COVID-19) has placed huge strains on health and social care systems and resources globally. The significance of infection prevention and control through measures such as hand hygiene, social distancing and self-isolation have now been emphasized at a societal level. It is important to consider the short and longer-term consequences that COVID-19 may have on antimicrobial use and drug-resistant infections. A concerning potential consequence of the COVID-19 pandemic is the long-term propagation of antimicrobial resistance (AMR) in the acute care setting, resulting from increased patient exposure to antimicrobials, often suboptimally or inappropriately used ${ }^{1}$. However, the simultaneous disruption of routine care and access to health care in acute and community settings for the non-COVID-19 population could potentially reduce the overall use of antimicrobials. The wider implications of the pandemic for health and social care systems must be characterized, and data must be gathered to inform and update national strategies that address the long-term challenges of AMR while preserving access to effective drugs.

\section{Individual and organizational impact}

In the short-term, antimicrobials have several roles during the pandemic. First, agents are being explored in clinical trials as potential direct therapies for severe acute respiratory syndrome coronavirus 2 (SARS-CoV-2), such as azithromycin ${ }^{2}$ and antivirals, such as lopinavir and ritonavir $^{3}$, and remdesivir. However, trials have yet to conclusively show improved clinical outcomes compared with standard of care. Second, antimicrobials are commonly prescribed for the management of presumptive or confirmed bacterial co-infection directly related to COVID-19, co-occurring at the time of infection, or associated with health care, such as prolonged admission to critical care. Current evidence suggests that bacterial and fungal co-infection in patients with COVID-19 is low $^{4}$; yet, prescribing rates and use of broad-spectrum antimicrobial agents is high ${ }^{4}$.
Combined with a lack of rapid diagnostic and decision support tools to support the clinical management of COVID-19, increased unnecessary antimicrobial use occurs. Direct risks to the patient include side effects and adverse drug events. Increases in unnecessary antimicrobial use potentiates future risk of AMR through driving selection of multidrug resistant (MDR) organisms and carbapenemase-producing Enterobacteriaceae. In parallel and in contrast, many routine care services in hospitals have been disrupted: non-COVID-19 patients may have not received elective care nor been exposed to antimicrobials.

Meanwhile, the impact of the pandemic on antimicrobial prescribing and AMR in the community remains largely unknown. Potential patient avoidance of health services owing self-isolation or an inability to access health care owing to service provision changes may limit demand but also restrict access to necessary antimicrobials. Sustained public campaigns on the role of antibiotics in viral infections as well as the increased use of telemedicine may also reduce antimicrobial prescribing and AMR, but this is yet to be described ${ }^{5}$.

At the health and social care level, organizations have had to rapidly respond and adapt to the challenges of the pandemic ${ }^{5}$. In addition to the service changes, some routine infection control practices may have been compromised, including isolation of patients with MDR organisms as well as the redeployment of diagnostics to focus on SARS-CoV-2 in place of routine screening and surveillance for resistant bacteria, other respiratory viruses and health-care-associated infections. However, emphasis on the role of hand hygiene may reduce transmission of AMR and diseases that would drive antibiotic use. Moreover, the pressure on finite resources, such as medications (including antimicrobials) and high volume needs such as personal protective equipment for patients with COVID-19 may deplete availability of resources for the prevention and management of other infectious diseases. This additional strain is likely to exert a substantial impact on the resource-limited settings and 
countries that have existing high burdens of endemic infectious diseases.

Following the initial global surge in COVID-19 cases, further surges are expected until control measures, such as vaccination, are introduced ${ }^{6}$. Health-care systems will need to maintain their ability to, for example, expand critical care capacity within short periods, while mitigating disruptions to other services and resources. It is vital that access to antimicrobials, stewardship and surveillance of AMR and health-care-associated infection systems remain in place, which may in turn require reorganization to ensure systems are robust enough to cope $\mathrm{e}^{1}$. Moreover, delays to elective surgery and cancer therapy will add pressure, potentially leading to extended health-care needs and increased antimicrobial use.

\section{Impact on national public health policies}

Consideration needs to be given to the impact of the COVID-19 pandemic on individual national action plans for AMR. With possible increases in antimicrobial usage in the acute care setting and disruption of public health programmes, such as national stewardship, infection control, tuberculosis management and childhood immunization, national action plans will have to be flexible. Vaccination has been identified as a potential key intervention for reducing antimicrobial usage and therefore drug-resistant infections ${ }^{4}$. There is a risk that the pandemic will lead to major disruptions to immunization services, with major long-term consequences, including secondary outbreaks of vaccine-preventable diseases ${ }^{7}$. The impact on control of MDR tuberculosis as a result of demands on health resources is likely to disproportionately impact low-income and middle-income countries, placing strain on fragile and overstretched health-care systems. It is vital that support and funding for these national programmes are maintained.

\section{Mitigation strategies}

To mitigate the potential short and long-term impact of COVID-19 on antimicrobial use and AMR, health and social care systems must act to ensure that knowledge and evidence are effectively gathered to rapidly shape understanding and inform action. Evaluation of the impact of the pandemic on antimicrobial usage, AMR and access to effective antimicrobial treatments is essential to support the development of mitigation strategies ${ }^{8}$. Clinical treatment guidelines to limit unnecessary antimicrobial exposure, measures to maintain routine surveillance of AMR and review of national policies that do not neglect essential public health programmes in tuberculosis control and immunization delivery are essential. Individual organizations and health-care systems must engage the wider public in a timely fashion, given the ongoing widespread awareness. These strategies need to be supported by responsive national and international action plans that embed AMR as a priority in the post-COVID-19 era.

At the national level, diagnostics and decision support should be developed to differentiate secondary bacterial infection during COVID-19. The role of specific biomarkers such as procalcitonin warrants further investigation. Linkage of data to artificial intelligence-driven decision support software may provide added benefit ${ }^{9}$. Antimicrobial stewardship should become an essential part of the individual clinical teams practice ${ }^{10}$. Broader stewardship engagement will promote wider adoption of interventions designed to optimize treatment and reduce inappropriate antimicrobial use. Within primary care, data are urgently required to evaluate the impact of COVID-19 on antimicrobial prescribing, antibiotic access and clinical outcomes of infection. Novel approaches to stewardship and optimizing prescribing including telemedicine must be explored ${ }^{5}$. At the wider health-care level, providers must share experiences and collaborate internationally. Rapid progress has been made globally in basic sciences, epidemiology, diagnostics and clinical research in tackling COVID-19. There is also a societal opportunity to capitalize on the experience and learning from the pandemic to improve existing infection control and to sustain the raised awareness of the importance of vaccines and effective antimicrobial therapy. Coordination at the national level is required to gather evidence on the complex consequences of COVID-19 to inform necessary actions to reduce the potential longer-term impact on AMR and access to effective antimicrobials.

1. Rawson, T. M. et al. COVID-19 and the potential long term impact on antimicrobial resistance. J. Antimicrob. Chemother. https:/ doi.org/10.1093/jac/dkaa194 (2020).

2. Gautret, P. et al. Hydroxychloroquine and azithromycin as a treatment of COVID-19: results of an open-label non-randomized clinical trial. Int. J. Antimicrob. Agents https://doi.org/10.1016/ j.ijantimicag.2020.105949 (2020)

3. Cao, B. et al. A trial of lopinavir-ritonavir in adults hospitalized with severe Covid-19. N. Engl. J. Med. 382, 1787-1799 (2020).

4. Rawson, T. M. et al. Bacterial and fungal co-infection in individuals with coronavirus: a rapid review to support COVID-19 antimicrobial prescribing. Clin. Infect. Dis. https://doi.org/10.1093/cid/ciaa530 (2020).

5. Hollander, J. E. \& Carr, B. G. Virtually perfect? Telemedicine for Covid-19. N. Engl. J. Med. 382, 1679-1681 (2020).

6. Ferguson, N. M. et al. Impact of non-pharmaceutical interventions (NPIs) to reduce COVID-19 mortality and healthcare demand. https://doi.org/10.25561/77482 (2020).

7. Santoli, J. M. et al. Effects of the COVID-19 pandemic on routine pediatric vaccine ordering and administration - United States, 2020. MMWR. Morb. Mortal. Wkly Rep. 69, 591-593 (2020)

8. Sharland, M. et al. Classifying antibiotics in the WHO Essential Medicines List for optimal use-be AWaRe. Lancet Infect. Dis. 18, 18-20 (2018).

9. Rawson, T. M. et al. A real-world evaluation of a Case-Based Reasoning algorithm to support antimicrobial prescribing decisions in acute care. Clin. Infect. Dis. https://doi.org/10.1093/cid/ciaa383 (2020).

10. Rawson, T. M., Moore, L. S. P. Gilchrist, M. J. \& Holmes, A. H. Antimicrobial stewardship: are we failing in cross-specialty clinical engagement? J. Antimicrob. Chemother. 71, 554-559 (2015).

\section{Acknowledgements}

The authors acknowledge funding from National Institute for Health Research. R.A. acknowledges funding from City, University of London, Division of Health Sciences.

\section{Competing interests}

L.S.P.M. has consulted for DNAelectronics (2015-2018), Dairy Crest (2017-2018), Umovis Lab (2020) and Pfizer (2018-2020), received speaker fees from Profile Pharma (2018), received research grants from CW + Charity (2018-2019) and Leo Pharma (2016), and educational support from Eumedica (2016-2018). The other authors declare no competing interests. 